\title{
ANÁLISIS DE LOS CONFLICTOS SOCIO-AMBIENTALES EN LOS PAÍSES ANDINOS UTILIZANDO EL ATLAS GLOBAL DE JUSTICIA AMBIENTAL COMO HERRAMIENTA ${ }^{1}$
}

por ZUZANA LHOTÁKOVÁ

(Universidad Carolina, Praga)

\begin{abstract}
Resumen
El trabajo analiza los conflictos socio-ambientales en tres países andinos: Perú, Ecuador y Bolivia, que comparten gran riqueza de recursos, alta biodiversidad y una parte significativa de la población indígena. Como herramienta se utilizó el Atlas Global de Justicia Ambiental, una plataforma que reúne información sobre más de 2.000 conflictos ecológico-distributivos en todo el mundo.
\end{abstract}

Palabras clave: justicia ambiental; medioambiente; países andinos; conflicto socio-ambiental

\section{Summary}

The article entitled The analysis of socio-environmental conflicts in Andean countries: The Global Atlas of Environmental Justice as a toolb analyses the socio-environmental conflicts in three selected Andean countries: Peru, Bolivia and Ecuador, which are characterized by natural resources abundance, high biodiversity and a significant share of indigenous population. The Global Atlas of Environmental Justice, a public platform mapping more than 2.000 socio-environmental conflicts, was used as a tool.

Key words: environmental justice; environment; Andean countries; socio-environmental conflicts

\section{Introducción}

La creciente economía causa una enorme presión con la extracción de recursos naturales, de la que surgen los problemas de contaminación y daños a los ecosistemas. El daño hacia el medio ambiente afecta negativamente la calidad de la vida humana e incluso produce conflictos sociales. El objetivo es analizar qué rasgos comunes y no comunes muestran los conflictos socio-ambientales en tres países andinos - Perú, Ecuador y Bolivia. Los tres países comparten gran riqueza de recursos, ecosistemas con alta biodiversidad y una parte significativa de la población rural e indígena la cual es la más dependiente de dichos ecosistemas. Por otro lado hay diferencias entre las legislaciones ambientales de estos países. Como herramienta se utilizó el Atlas Global de Justicia Ambiental (https://ejatlas.org), una plataforma que reúne información sobre más de 2.000 conflictos ecológico-distributivos en todo el mundo.

\section{Desarrollo económico y sus efectos ambientales}

El modelo contemporáneo del desarrollo global cuenta cada vez con más producción y consumo, y por otro lado la proporción del reciclaje de las materias primas sigue siendo muy baja. La creciente economía mundial basada en la producción perpetua causa una enorme presión en la extracción de recursos naturales, tanto si son renovables

1 La investigación fue realizada en el marco del proyecto de la Universidad Carolina, Facultad de Filosofía y Letras, SVV-260422. 
como convencionales. De esta extracción excesiva surgen problemas de contaminación del aire, contaminación del agua y del suelo, acumulación de deshechos o daños a los ecosistemas naturales. Las fronteras de la extracción de muchas materias primas, como el petróleo y los metales, están avanzando incluso hacia los ecosistemas que tienen el papel principal en el mantenimiento del clima global o el ciclo del agua. Además la extracción, la agricultura intensiva y las plantaciones de monocultivos amenazan a los ecosistemas naturales de alta biodiversidad. Aunque existe un concepto de servicios de los ecosistemas (Ecosystem services $^{2}$ ), que propone la valoración financiera de los ecosistemas naturales, los costes ambientales (por ejemplo la contaminación) no son incluidos en la contabilidad de la mayoría de las empresas y quedan fuera del punto de vista económica como las 'externalidades'. Al contrario, muchas opiniones o conceptos consideran que la naturaleza y la biodiversidad tienen valor por sí mismos, que no se puede expresar en dinero ${ }^{3}$. El daño al medio ambiente afecta de forma negativa la calidad de la vida humana en muchos aspectos, incluso la salud, el acceso al agua potable y la seguridad alimentaria. Sin embargo, en el mundo globalizado estos impactos negativos tanto como los beneficios, no son distribuidos igualmente entre la población y las regiones, lo que genera injusticias ambientales que a menudo provocan manifestaciones y conflictos sociales. En casos extremos estos conflictos desembocan en la migración forzada, el involuntario cambio de estilo de vida e incluso la muerte de gente durante las protestas públicas.

\section{Justicia Ambiental}

La idea de la justicia ambiental tiene sus raíces en los Estados Unidos al final de los años setenta ${ }^{4}$, cuando comenzaron las protestas públicas contra

2 Robert COSTANZA - Ralph D'ARGE - Rudolf DEGROOT - Stephen FARBER et al., "The Value of the World's Ecosystem Services and Natural Capital", Nature 387 (6630), London 1997, pp. 253-260.

3 Carlos VITERI GUALINGA, "Visión indígena del desarrollo en la Amazonía", Polis. Revista Latinoamericana 3, Poetro Mont 2002, [consultado 25 de septiembre de 2017], accesible de: http://polis.revues.org/7678.

4 Phil BROWN, "Race, Class, and Environmental Health: A Review and Systematization of el almacenamiento de residuos tóxicos, preferentemente cerca de los barrios poblados de gente de color. Las protestas aisladas se convirtieron en un movimiento global durante las siguientes décadas ${ }^{5}$. También se ha ampliado la variedad de los conflictos: además de los problemas con residuos tóxicos surgen más conflictos sobre recursos de agua, minería y conservación de la biodiversidad. El EJAtlas, el Atlas Global de Justicia Ambiental, es el resultado de una iniciativa colaborativa de un equipo internacional. Se trata de un inventario de los conflictos socio-ambientales de todo el mundo, de acceso al público en internet (https:// ejatlas.org) y según sus editores y autores debería servir como una nueva herramienta para los activistas, los académicos y las organizaciones ambientalistas. ${ }^{6}$ Ahora mismo el EJAtlas cuenta con más que 2000 conflictos socio-ambientales y, de estos, unos 680 se encuentran en Latinoamérica - lo que supone ser de un tercio de todos los conflictos incluidos en el EJAtlas. La utilización del EJAtlas también tiene potencial para fortalecer la colaboración entre los académicos, activistas, organizaciones no gubernamentales, administración pública y empresas lo que tiene el papel principal en la búsqueda de la sostenibilidad de la sociedad global.

\section{Ecuador, Perú y Bolivia - característica}

Para el análisis he elegido tres países andinos que comparten algunas características importantes. Supongo que precisamente estas características pueden tener influencia en el surgimiento y en la manera de tratar los conflictos socio-ambientales. Los tres países elegidos disponen de riqueza de recursos agotables (petróleo/gas natural, metales), ecosistemas de alta biodiversidad (la Amazonía) y ecosistemas importantes para el ciclo del agua (los páramos). En todos estos países hay una

the Literature", Environmental Research 69 (1), Amsterodam 1995, pp. 15-30

5 Joan MARTÍNEZ-ALIER - Leah TEMPER Daniela DEL BENE et al., "Is there a global environmental justice movement?", The Journal of Peasant Studies 43 (3), Abingdon 2016, pp. 731-755.

6 Leah TEMPER - Daniela DEL BENE - Joan MARTÍNEZ-ALIER, "Mapping the frontiers and front lines of global environmental justice: the EJAtlas", Journal of Political Ecology 22, Tucson 2015, pp. 255-278. 
significativa parte de la población rural e indígena que con su estilo de vida depende de dichos ecosistemas.

Según el EJAtlas, los conflictos están clasificados en diez categorías: contaminación nuclear, minería, manejo de residuos tóxicos, biomasa y conflictos en el uso del suelo, combustibles fósiles, manejo del agua, infraestructura, turismo y recreación, conservación de la biodiversidad, industrias. ${ }^{7}$ Actualmente en Perú hay 78 conflictos, en Ecuador 61 conflictos y en Bolivia 40. ${ }^{8}$ El marco temporal en el que han empezado los conflictos abarca casi las tres últimas décadas y por eso hay que considerar la situación política y económica de los países. Sirva de ejemplo el desarrollo de la normativa ambiental indicada más adelante.

Desde los años ochenta hasta 2006 el Ecuador seguía una agenda neoliberal incluso con ajustes de instituciones financieras internacionales como el Banco mundial. La economía estaba basada en la extracción petrolera. El país pasaba por una inquietud social con la movilización de los movimientos indígenas. Después de la reactivación del crecimiento económico acabó con el aumento de desigualdad social y la crisis financiera en 1999 , que más tarde resultó en la dolarización de la economía. La normativa ambiental era rudimentaria hasta 1999. Desde 2006, con el presidente Correa, la agenda se transformó en post-neoliberal con la nueva Constitución (2008), que incluye el desarrollo sostenible, buen vivir - sumak kawsay, conservación y derechos de naturaleza. Sin embargo la matriz productiva sigue siendo extractivista.

En los años ochenta Perú sufrió una fuerte crisis económica por la hiperinflación, alto desempleo y otros altibajos de la economía. Lo que se refiere a la normativa ambiental, hasta finales de los años ochenta el Perú contaba con una legislación ambiental eminentemente sectorial y dispersa que carecía de un marco integrador. Los noventa destacaron con la apertura de la economía gracias a una liberalización agresiva, la privatización del sector extractivo (minería, hydrocarburos) y las inversiones extranjeras transnacionales. Aunque en Perú no había tanto cambio en la orientación económica, como en Ecuador y Bolivia, los que seguían en el régimen (neo)liberal, al menos la normativa ambiental se ha reforzado desde 2005.

Ibid.

8 Actualización última: 29 de mayo de 2017, accesible de: http://ejatlas.org.
Sin embargo la minería y la refinación de minerales, extracción de petróleo y gas natural son las principales industrias de hoy en día.

En Bolivia la situación es más parecida a Ecuador. Las reformas neoliberales de los ochenta contaban con la privatización de industrias estatales incluso sectores de hidrocarburos y préstamos del Banco mundial. En el 2003 sufrió el país unas protestas contra exportaciones de gas a EE.UU. (conocida como la guerra del gas) con la fuerte movilización popular y varias renuncias presidenciales forzadas. Desde 2005, con el presidente Morales, se ha nacionalizado de nuevo el sector de los hidrocarburos. La nueva constitución de 2009 declara a Bolivia como un estado plurinacional, igualmente como la ecuatoriana incluye ideas de vivir bien y reconoce la naturaleza como la Madre Tierra. Tal como en Perú y Ecuador la economía boliviana sigue más extractivista y desarrollista que ambientalista.

\section{Normativa ambiental en los países elegidos}

Por lo que se refiere a la legislación ambiental existen diferencias entre los países investigados. Por ejemplo, la protección del medioambiente se apoya en documentos de diferente razón jurídica: en Ecuador los derechos de la naturaleza están incluidos en la Constitución del Estado; en Bolivia la protección del medioambiente se apoya en la Ley de derechos de la Madre Tierra y en Perú en el Código del Medio Ambiente y los Recursos Naturales y La Ley General del Ambiente. Al final de nuestro análisis veremos la normativa ambiental en cada un país con más detalle.

En Ecuador, la actual Constitución del estado está vigente desde el año 2008 y abarca dos perspectivas para el respeto del medio ambiente. La primera está de acuerdo con el punto de vista antropocéntrico y coincide con los principios del derecho europeo. En este sentido la Constitución garantiza a los ciudadanos el derecho "a vivir en un ambiente sano y ecológicamente equilibrado, que garantice la sostenibilidad y el buen vivir, sumak kawsay", 9 o se exige la conservación de la biodiversidad y regeneración de los ecosistemas. ${ }^{10}$ La segunda perspectiva se basa en la actitud holística y biocéntrica de las cosmovisiones indígenas.

9 Constitución del Ecuador 2008, Art. 14., Capítulo segundo, Título I.

10 Constitución del Ecuador 2008, Art. 395., Capítulo segundo, Título VII. 
La naturaleza o Pacha Mama viene definida como un sujeto de los derechos constitucionales, ${ }^{11}$ lo que es una situación muy singular en la normativa ambiental en todo el mundo.

En Perú, el marco constitucional reconoce el derecho fundamental de las personas a gozar de un ambiente saludable, el marco legislativo general asegura el Código del Medio Ambiente y los Recursos Naturales desde 1990 y desde 2005 La Ley General del Ambiente (Ley No 28611). ${ }^{12}$ En Perú, la normativa es más antropocéntrica, lo que significa que el ser humano está en el centro y la naturaleza viene definida como medio ambiente y ecosistemas, y siempre como objeto de los derechos de los ciudadanos.

En Bolivia, la Constitución del Estado de forma similar a la peruana garantiza a las personas vivir en un ambiente saludable, protegido y equilibrado. Además, en el año 2010 se aprobó la Ley de derechos de la Madre Tierra. Desde el punto de vista jurídico la Madre Tierra viene definida como un sujeto colectivo de interés público. La perspectiva de esta ley es más holística y se acerca más al caso ecuatoriano - la Madre Tierra está entendida como un sistema viviente y los humanos forman parte de ella junto con otros seres vivos..$^{13}$

\section{Categorización de conflictos socio-ambientales según EJAtlas}

Tres categorías de conflictos que son más frecuentes en estos tres países: conflictos de minería, de combustibles fósiles / justicia climática y del manejo del agua. Los conflictos de minería destacan en Perú ya que hay tres veces más conflictos de minería en contraste con Ecuador y Bolivia (Figura 1). Los conflictos de minería representan la categoría de conflictos frecuentemente

11 Constitución del Ecuador 2008, Art. 11., Capítulo primero, Título II; Art. 71-74., Capítulo séptimo, Título II, 2008.

12 La Ley General del Ambiente, [consultado 29 de mayo de 2017] accesible de: http://www .legislacionambientalspda.org.pe/index.php ?option=com_content $\&$ view $=$ article $\&$ id $=$ $6 \&$ Itemid $=4714$.

13 Ley 071, 2010 capítulo ii, madre tierra, definición y carácter, Art. 3.-6. 2010. analizada, ${ }^{14}$ incluso utilizando el mismo EJAtlas. ${ }^{15}$ Los conflictos de combustibles fósiles, justicia climática y energía le siguen en segundo lugar. En este ensayo voy a enfocarme en esta categoría de los conflictos y la lista de ellos se encuentra en al Apéndice con identificación según el país (E-Ecuador, P-Perú, B-Bolivia y numeración cronológica). Porque se trata de la extracción de los recursos no renovables como en el caso de la minería, espero que existan rasgos comunes en su desarrollo asimismo en la motivación de resistencia. Los conflictos sobre el manejo del agua aparecen en tercer lugar - se trata a menudo de construcción de represas y hidroeléctricas, contaminación del agua y privatización del agua (Ecuador - Guayaquil 2001, Bolivia - Cochabamba 1999, La Paz 2005). En estos tres países los conflictos de biomasa y el uso del suelo le siguen en abundancia en el cuarto lugar.

\section{Conflictos por Combustibles fósiles / justicia climática - descripción.}

En Ecuador y Perú prevalecen los conflictos de explorar y extraer petróleo. En Bolivia se trata más bien de la combinación producida por la exploración del petróleo y gas natural. La diversificación depende del recurso pero, en los rasgos, los conflictos tienen mucho en común como será presentado más adelante. La mayoría de los conflictos ocurre en la zona de las tierras bajas en el este del país - Amazonía y Chaco en Bolivia, por la ubicación de las reservas de dichos recursos (petróleo/gas). Una parte de los conflictos tiene origen en las áreas donde la extracción petrolera ya se desarrollaba desde antes (E1, E5, P1, P2, B1, B2), la otra parte coincide con proyectos de exploración e intentos de ampliación de la frontera extractiva petrolera (E4, E16, P8, B4). Sin pocas excepciones, la población rural es la más afectada y muchas veces son los pueblos indígenas los que no comparten la moderna idea de naturaleza como un objeto de dominación y explotación (Vallejo 2014). Por eso es lógico que, entre los grupos que

14 Marta CONDE, "Resistance to Mining. A Review”, Ecological Economics 132, Amsterodam 2017, pp. 80-90.

15 Begüm ÖZKAYNAK - Beatriz RODRÍGUEZLABAJOS - Cem İSKENDER AYDIN, "Towards environmental justice success in mining conflicts: An empirical investigation", EJOLT Report 14, Barcelona 2015, p. 96 


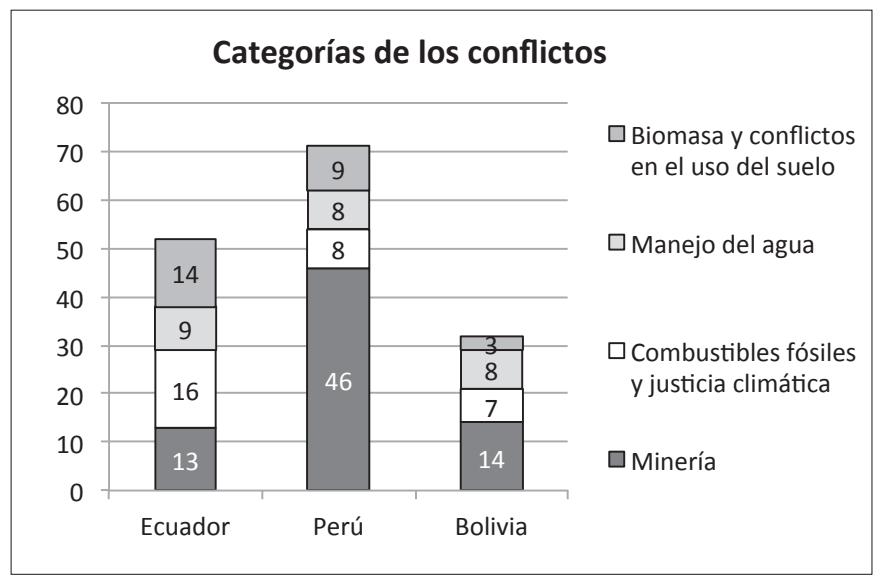

Figura 1: Categorías de conflictos socio-ambientales en Ecuador, Perú y Bolivia. Se presentan solo las cuatro categorías con más abundancia, las categorías pendientes no cuentan más de tres conflictos en los países analizados. Recurso de datos: EJAtlas, https://ejatlas.org. Elaboración propia (mayo 2017).

se movilizan para protestar, casi siempre participan las nacionalidades indígenas y comunidades tradicionales, lo que es válido para todos países investigados: Ecuador 12 de 16 casos, Perú 7 de 8 casos y Bolivia todos los casos, 7 (Apéndice - Tabla 1 y 2 ).

\section{Objetivos}

El objetivo principal es analizar qué rasgos comunes y no comunes muestran los conflictos socio-ambientales en los tres países escogidos - Perú, Ecuador y Bolivia. Como objetivo secundario, intentaré destacar los puntos fuertes y débiles sobre la utilización del EJAtlas como herramienta en este caso en concreto. Para cumplir el objetivo principal he formulado las siguientes preguntas:

1. ¿Existe una relación entre la motivación de la resistencia contra la extracción de combustibles fósiles y la perspectiva de la normativa ambiental (antropocéntrica/holística)?

2. ¿Existe una diferencia entre los países respecto a la percepción del éxito de justicia ambiental dentro los conflictos de combustibles fósiles?

\section{Análisis \\ 1. ¿Existe una relación entre la motivación de la resistencia contra la extracción de combustibles fósiles y la perspectiva de la normativa ambiental (antropocéntrica/holística)?}

Supongamos que en los países que en su normativa declaran derechos de la naturaleza y la Madre Tierra como en Ecuador y Bolivia sea más frecuente que la motivación de resistencia y los argumentos contra la extracción se apoyen en la reivindicación de estos derechos. Por otro lado hemos visto que incluso en Perú, donde este tipo de normativa no existe, los grupos indígenas se movilizan frecuentemente contra la extracción de hidrocarburos. Este hecho puede basarse en el diferente concepto de naturaleza en las cosmovisiones indígenas, que reconocen otros significados de ella, además del ambiente y el recurso. Examinamos si podemos identificar este hecho en desarrollo y la solución de los conflictos socio-ambientales.

Con respeto a las formas de movilización y resistencia, existe una variedad amplia en todos los países, desde campañas públicas y mediáticas, marchas, peticiones, asambleas hasta la actividad jurídica. Podemos encontrar notable diferencia entre los países en número de conflictos por combustibles fósiles que incluyen demandas jurídicas: Ecuador 11 de 16 casos, Perú 3 de 8 casos y Bolivia ninguno de 7 casos. Exploramos un poco la idea de si las demandas incluyen los derechos de la naturaleza y la Madre Tierra. Tres de los once casos ecuatorianos los reivindican, igualmente dos los tres casos peruanos. Además, un conflicto en cada país tiene la motivación en los derechos de la Madre Tierra pero sin demanda judicial. 


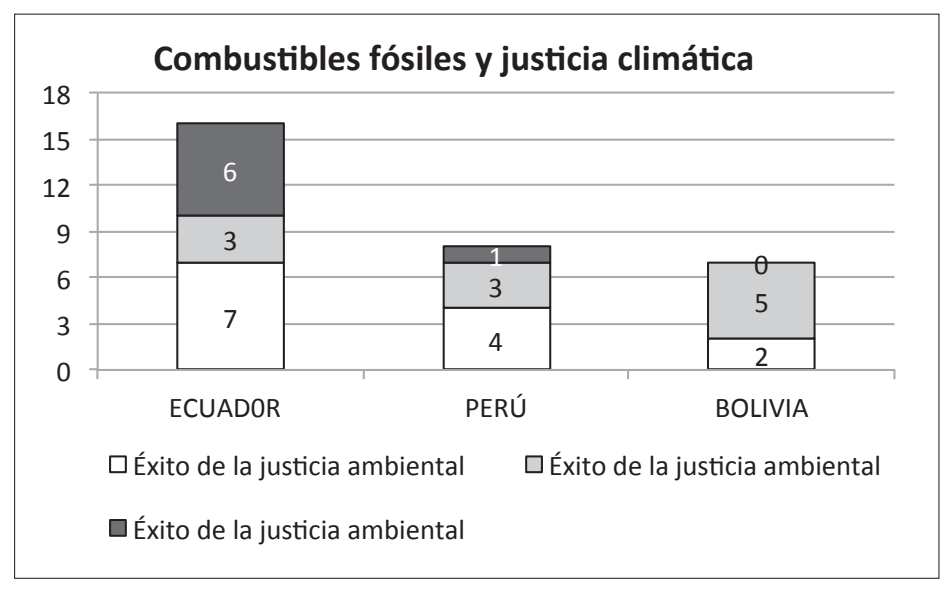

Figura 2: Resultados de los conflictos desde punto de vista de la justicia ambiental. Recurso de datos: EJAtlas, https://ejatlas.org. Elaboración propia (mayo 2017).

Como se afirmó arriba, la movilización indígena, la otra fuerte herramienta legal en los conflictos son los derechos de los pueblos indígenas para el control de sus propias instituciones y formas de vida y de su desarrollo económico y a mantener y fortalecer sus identidades, que reconocen los códigos legales nacionales, incluso declarados por las normas internacionales como el Convenio núm. 169 de la OIT del año 1989. En muchos casos, la movilización indígena proviene de la ausencia de una consulta previa sobre la gestión de los territorios indígenas, lo que también garantizan dichas normativas nacionales e internacionales.

Para concluir la relación entre la motivación de la resistencia y la perspectiva de la normativa ambiental no es tan estrecha. En primer lugar los argumentos contra los proyectos de extracción y exploración son potencial amenaza y pérdida del estilo de vida tradicional y la contaminación del agua, el suelo y consecuentemente los impactos negativos para la salud. Aunque la motivación de proteger el ambiente está presente, no figura tan menudo en forma de reivindicación de los derechos de la naturaleza / Madre Tierra. Deseo subrayar que el reconocimiento de los derechos de la naturaleza está incluido en la normativa de Ecuador y Bolivia no más que una década ya que, en la práctica, todavía no se ha logrado asentar y falta más tiempo para convertir esos pensamientos en una práctica cultural.

2. ¿Existe una diferencia entre los países respecto a la percepción del éxito de justicia ambiental dentro los conflictos de combustibles fósiles?

Cada ficha del conflicto documentado por el EJAtlas incluye una valoración de si los grupos movilizados y las comunidades afectadas evalúan el resultado del conflicto como éxito o fracaso de la justicia ambiental. La valoración es frecuentemente ambigua (opción "no seguro") y siempre está complementada con una explicación. Empezamos con la valoración cuantitativa simple de los conflictos por combustibles fósiles y justicia climática y veremos que la proporción de casos considerados como exitosos y fracasados es más alta en Ecuador (6:7) que en Perú (1:4). En Bolivia no existe ningún caso con resultado considerado como exitoso e igualmente la proporción como el número absoluto de los resultados ambiguos es la más alta de todos los países investigados (Figura 2). Como resultado, parece que el Ecuador destaca no solo en reivindicación de la justicia ambiental sino también en la percepción de conseguirla.

Todos los conflictos (Ecuador: E2, E6, E7, E8, E10, E11; Perú: P3) evaluados como éxito de la justicia ambiental tienen en común lo que se trata de daños a la salud y la grave contaminación del ambiente como resultado de actividades extractivistas anteriores. Siempre el resultado se logró por una demanda judicial y la decisión de la corte en el favor de las comunidades y ciudadanos afectados. Las compañías petroleras (no solo transnacionales sino también las nacionales) fueron obligadas a limpiar el medio ambiente, remediar 
los daños y pagar una compensación financiera a las comunidades. Podemos entender estos casos como precedente importantes para el futuro. Pero como veremos más adelante, la compensación financiera no siempre es conmensurable con los efectos negativos sociales y ambientales. De los resultados exitosos solo un caso ecuatoriano (E2) puso como argumento los derechos de la naturaleza (en el sentido de la Constitución de 2008), otros dos reivindicaron los derechos garantizados por la Ley de gestión ambiental y la Ley de prevención y control de la contaminación ambiental (E7, E10, E11). Dos casos más ecuatorianos (E6, E8) reivindicaron los derechos de los pueblos indígenas según el Convenio 169 de OIT. Por lo que se refiere a Perú, el acuerdo se logró al llegar a la corte estadounidense en 2015. Aunque por entonces la valoración del resultado fue positiva, la conflictividad en la zona se reavivó en diciembre del 2016 , cuando se registró un derrame, cuya responsabilidad recayó en la transnacional canadiense Pacific Exploration \& Production, que actualmente opera el lote 192. El conflicto sigue negociando el monto de la compensación por el uso de las tierras. ${ }^{16}$

La valoración negativa de los resultados del conflicto en Ecuador se basa sobre todo en el incumplimiento de lo ofrecido por las empresas petroleras y el gobierno (E3, E4, E14). De igual manera las comunidades afectadas perciben incumplimiento de sus derechos constitucionales para la consulta previa a lo que se refiere a la explotación de recursos no renovables que se encuentren en sus tierras ${ }^{17}$ (E9, E13, E14, E16). La situación en Perú es similar ya que algunos proyectos continúan a pesar de la reclamación de derechos ancestrales por comunidades indígenas (P6). Otros siguen en construcción y operación promovidos por las autoridades nacionales (P1, P7) y grupos financieros internacionales como el Banco Interamericano de Desarrollo (P2). En Bolivia la evaluación de injusticia ambiental proviene en primer lugar del incumplimiento de la recuperación de las áreas afectadas (B1, B5). En segundo lugar el fraccionamiento de las comunidades que resultaron de los problemas por el manejo de la compensación económica y la

16 Consultado 24 de septiembre de 2017, accesible de: https://actualidad.rt.com/actualidad /237018-indigenas-peru-ocupan-lote-192.

17 Constitución del Ecuador 2008, Art. 57., Capítulo cuatro, Título II, 2008. corrupción para silenciar el conflicto es también la razón para valorar negativamente el resultado.

La valoración ambigua de los resultados del conflicto provienen de varias razones y muchas veces quedan casos abiertos. En Ecuador se trata de dos casos de éxitos parciales como la salvación de algunos sitios arqueológicos (E1) y compensación financiera (E2). En el caso E1 el oleoducto pasa por el Parque Nacional Machalilla y el riesgo de contaminación permanece. De igual modo las tareas de exploración en áreas protegidas siguen en Bolivia (B3). El caso E2 es el conocido proceso legal de Chevron-Texaco en el que los daños ambientales son inconmensurables con cualquiera compensación financiera, lo que es la misma razón en valorar el resultado de derrames y contaminación petrolera del boliviano río Desaguadero (B2). En dos casos peruanos (P4, P5) siguen las actividades explorativas, en el tercer caso (P8) también se dio la autorización a la empresa estatal Pluspetrol para continuar con su plan de exploración. ${ }^{18}$ La división de las comunidades afectadas por cooptación por parte de gobiernos locales (B4) muestra el parecido patrón como mencionado antes (B1 y B5). También el futuro de los proyectos explorativos y extractivos continúan abiertos (B6) a pesar de resultados negativos al contrario de las expectativas (B4) ${ }^{19}$

En síntesis, la percepción del éxito de justicia ambiental es parecida en los tres países investigados, lo que demuestran sobre todo las semejanzas entre los razones de valoración negativa y ambigua. El mayor contraste surgió entre Ecuador y Bolivia: aunque ambos países disponen de derechos de la Madre Tierra en su normativa, su reivindicación prevalece en los casos ecuatorianos.

\section{El Atlas Global de Justicia Ambiental como herramienta}

Con respecto al trabajo con el EJAtlas sirvió de excelente herramienta para la rápida clasificación de los conflictos en el ámbito de interés. El objetivo de este ensayo fue obtener una idea

18 Consultado 24 de septiembre de 2017, accesible de: http://larepublica.pe/impresa/economia /800719-pluspetrol-invertira-hasta-us-400 -mllns-en-lote-108.

19 Consultado 29 de mayo de 2017, accesible de: http://www.paginasiete.bo/economia/2016 /3/24/lliquimuni-promociono-como-proyecto -estrella-pero-fracaso-90878.html. 
principal sobre los tipos de conflictos socio-ambientales en los países andinos mencionados. Hay que destacar la ventaja de que el EJAtlas presenta no solo los casos conocidos, como por ejemplo la extracción del petróleo en el Parque Nacional Yasuní - ITT, sino también conflictos de significado local y nacional.

Por un lado el EJAtlas permite la comparación de varios conflictos sin necesidad de la investigación de campo propia. Los autores del EJAtlas claramente definen que consideran los tipos de casos de resistencia como un conflicto socio-ambiental, ${ }^{20}$ declaran el proceso de control de la calidad y verificación de los datos e incluso la observación en el campo, existen algunas dudas sobre la credibilidad del EJAtlas. Por ejemplo, hubo casos indicados en el EJAtlas como despojos de tierra por construcción de las plantas eólicas en Oaxaca, pero según la comunicación personal (prof. Miguel Martínez González, Universidad del Mar, Mexico) $)^{21}$ son dudosas. Deseo subrayar que los mismos autores del EJAtlas lo entienden como un proyecto híbrido que está difuminando las fronteras convencionales entre la investigación científica y la participación de la sociedad civil incluyendo el activismo ${ }^{22}$ y ponen énfasis en el concepto de coo-diseño -colaboración de los académicos y los movimientos sociales- en la investigación en el ámbito de ciencias sociales. ${ }^{23}$

Este breve ensayo sin posibilidad de comprobar los conflictos analizados en el campo, trabaja casi exclusivamente con los datos del EJAtlas. A pesar de la consciencia de que con algunos casos ser a deseable buscar más recursos. Para ejecutar una investigación más profunda sería ideal combinar los recursos académicos con investigación de campo, entrevistas y reportes de los activistas como por ejemplo en un inventario

20 Véanse los criterios en Temper et al. 2015.

21 Comentarios y crítica de la ponencia de la autora sobre el mismo tema en IV Congreso Latinoamericano y Caribeño de Ciencias Sociales, Salamanca, España, 18 de julio de 2017.

22 Leah TEMPER - Daniela DEL BENE - Joan MARTÍNEZ-ALIER, "Mapping the frontiers", pp. 255-278.

23 Leah TEMPER - Daniela DEL BENE, "Transforming Knowledge Creation for Environmental and Epistemic Justice", Current Opinion in Environmental Sustainability 20, Amsterodam 2016, pp. 41-49. de la resistencia socio-ambiental en Ecuador, ${ }^{24}$ de ese modo, el EJAtlas servir como un recurso complementario. Sin embargo, tomando en cuenta algunas limitaciones, el EJAtlas puede servir como punto de partida para diseñar una investigación más profunda.

También es imprescindible trabajar con la temporalidad de los conflictos. Para ilustrar mejor consideremos la última comparación de los conflictos valorados por ambigüedad - la valoración fue basada en la incertidumbre del futuro desarrollo y por eso fue imprescindible actualizar los datos. Con el futuro intento de una comparación cualitativa más profunda. Habrá que tomar en consideración que el tipo y detalles de los conflictos también dependen de la actividad y participación de los científicos, voluntarios y activistas en cada país y región en concreto.

(Escrito en español por la autora)

\section{BIBLIOGRAFÍA}

http://ejatlas.org

BROWN, Phil, "Race, Class, and Environmental Health: A Review and Systematization of the Literature", Environmental Research 69 (1), Amsterdam: Elsevier, 1995, pp. 15-30.

COSTANZA, Robert, D’ARGE, Ralph, DEGROOT, Rudolf, et al. "The Value of the World's Ecosystem Services and Natural Capital", Nature 387 (6630), London: Springer Nature, 1997, pp. 253-260.

CONDE, Marta, "Resistance to Mining: A Review", Ecological Economics 13, Amsterdam: Elsevier, 2017, pp. 80-90.

LATORRE, Sara, N. FARRELL, Katharine, MARTÍNEZ-ALIER, Joan, "The Commodification of Nature and Socio-Environmental Resistance in Ecuador: AnInventory of Accumulation by Dispossession Cases, 1980-2013", Ecological Economics 116, Amsterdam: E1sevier, 2015, pp. 58-69.

24 Sara LATORRE - Katharine N. FARRELL Joan MARTÍNEZ-ALIER, "The Commodification of Nature and Socio-Environmental Resistance in Ecuador: An Inventory of Accumulation by Dispossession Cases, 1980-2013", Ecological Economics 116, Amsterodam 2015, pp. 58-69. 
MARTÍNEZ-ALIER, Joan, TEMPER, Leah, DEL BENE, Daniela, SCHEIDEL, Arnim, "Is there a global environmental justice movement?", The Journal of Peasant Studies 43(3), Abingdon: Taylor and Francis, 2016, pp. 731-755.

ÖZKAYNAK, Begüm, RODRIGUEZ LABAJOS, Beatriz, AYDIN, Cem İskender, YANEZ, Ivonne, GARIBAY, Claudio, "Towards environmental justice success in mining conflicts: An empirical investigation", EJOLT Report 14, Barcelona: Universitat Autònoma de Barcelona, 2015, 96 p.

TEMPER, Leah, DEL BENE, Daniela, MARTÍNEZ-ALIER, Joan "Mapping the frontiers and front lines of global environmental justice: the EJAtlas", Journal of Political Ecology 22, Tucson: University of Arizona, 2015, pp. 255-278.

TEMPER, Leah, DEL BENE, Daniela, “Transforming Knowledge Creation for Environmental and Epistemic Justice", Current Opinion in Environmental Sustainability 20, Amsterdam: Elsevier, 2016, pp. 41-49.

VITERI GUALINGA, Carlos, "Visión indígena del desarrollo en la Amazonía", [on-line] Polis. Revista Latinoamericana 3, Puerto Mont: Universidad de Los Lagos, 2002 [consultado 25 de septiembre de 2017]. Accesible de: http://polis.revues.org/7678. 


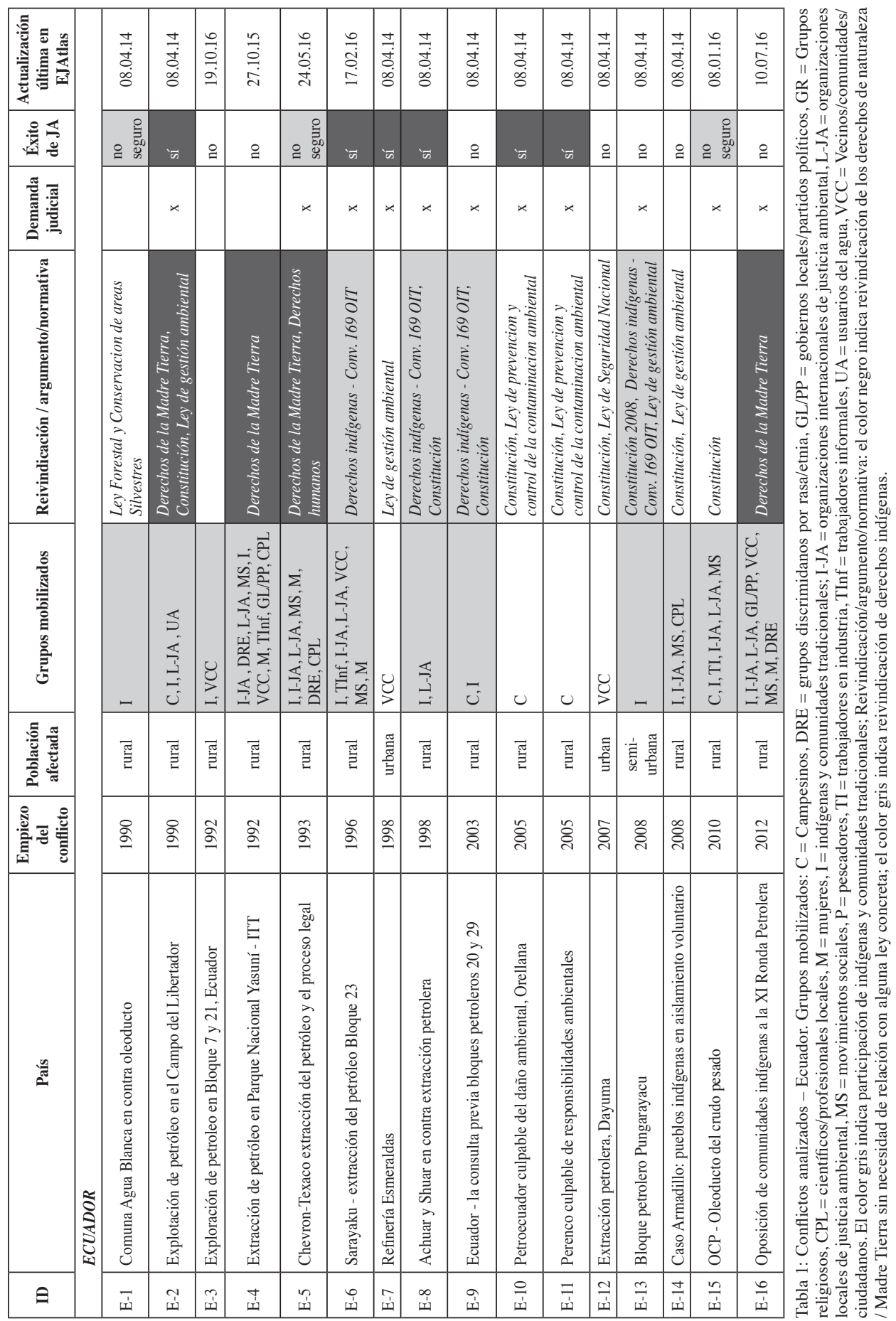




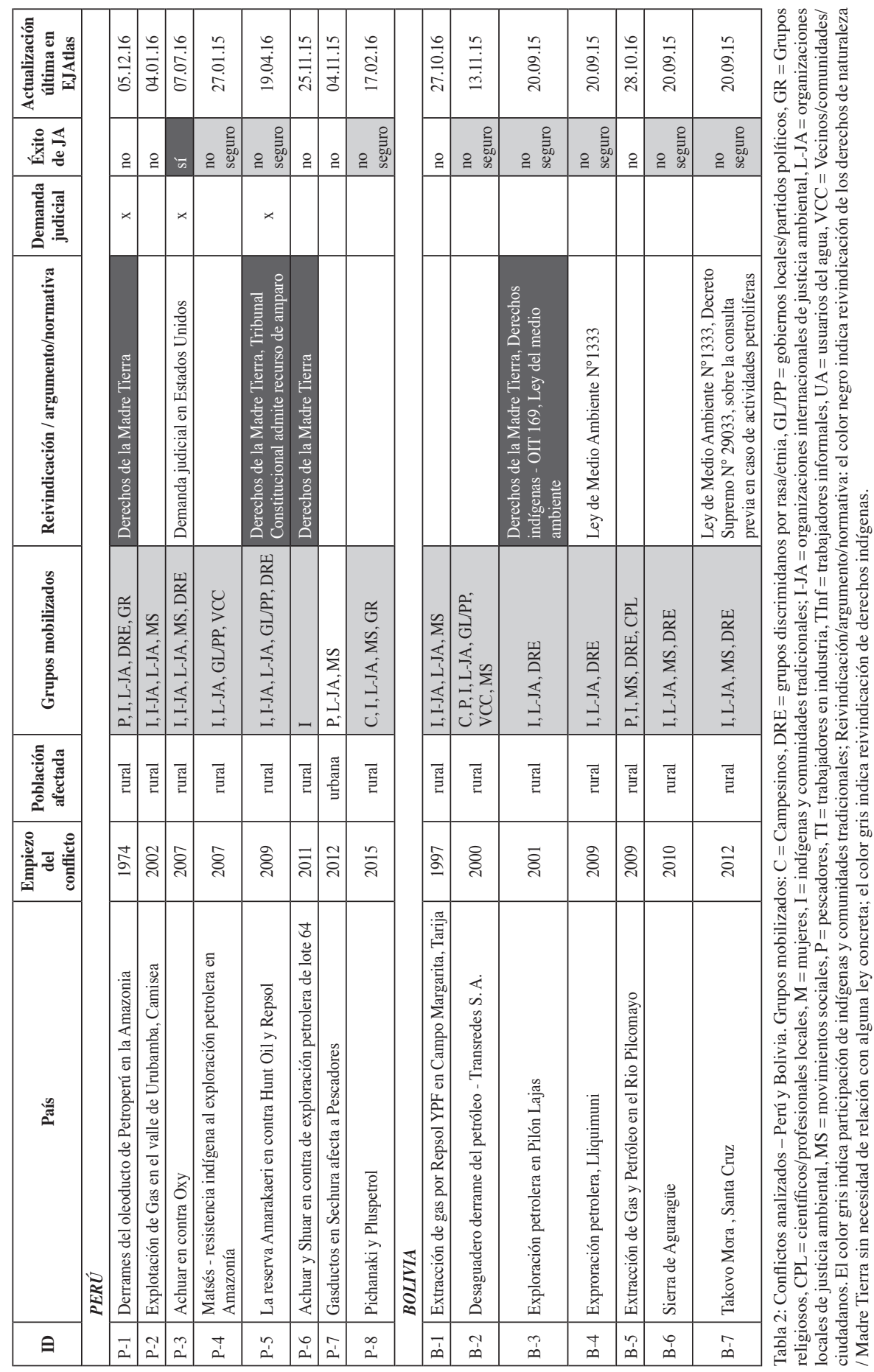




\section{Información breve sobre la autora}

Zuzana Lhotáková es el estudiante posgrado en el Centro de Estudios Ibero-Americanos, Facultad de Filosofía y Letras, Universidad Carolina, Praga, República Checa. El área de interés es la protección del medioambiente y las políticas del desarrollo sostenible en países andinos y los conflictos socio-ambientales.

Correo electrónico: zuza.lhotak@seznam.cz

\section{APÉNDICE}

Analizados conflictos de combustibles fósiles y justicia climática. Recurso de datos: EJAtlas, https://ejatlas.org. Elaboración propia (mayo 2017). 